\title{
Changes of functional connectivity in the left frontoparietal network following aphasic stroke
}

\author{
Dan Zhu ${ }^{1,2}$, Jingling Chang ${ }^{1}$, Sonya Freeman ${ }^{2}$, Zhongjian Tan $^{3}$, Juan Xiao ${ }^{1}$, Ying Gao ${ }^{1 *}$ and \\ Jian Kong ${ }^{*}$.
}

${ }^{1}$ Department of Neurology, Dongzhimen Hospital, Beijing University of Chinese Medicine, Beijing, China

2 Department of Psychiatry, Massachusetts General Hospital, Harvard Medical School, Boston, MA, USA

${ }^{3}$ Department of Radiology, Dongzhimen Hospital, Beijing University of Chinese Medicine, Beijing, China

Edited by:

Nim Tottenham, University of California Los Angeles, USA

\section{Reviewed by:}

Jesse Brown, University of

California Los Angeles, USA

Xilin Shen, Yale University, USA

*Correspondence:

Ying Gao, Dongzhimen Hospital, No. 5 Haiyuncang, Dongcheng

District, Beijing, 100700, China e-mail: gaoying973@126.com;

Jian Kong, Department of

Psychiatry, Massachusetts Genera Hospital, Harvard Medical School,

120 2nd Ave., Room 101C,

Charlestown, Boston, MA 02129,

USA

e-mail: kongj@nmr.mgh.harvard.edu

Language is an essential higher cognitive function supported by large-scale brain networks. In this study, we investigated functional connectivity changes in the left frontoparietal network (LFPN), a language-cognition related brain network in aphasic patients. We enrolled 13 aphasic patients who had undergone a stroke in the left hemisphere and age-, gender-, educational level-matched controls and analyzed the data by integrating independent component analysis (ICA) with a network connectivity analysis method. Resting state functional magnetic resonance imaging (fMRI) and clinical evaluation of language function were assessed at two stages: 1 and 2 months after stroke onset. We found reduced functional connectivity between the LFPN and the right middle frontal cortex, medial frontal cortex, and right inferior frontal cortex in aphasic patients as compared to controls. Correlation analysis showed that stronger functional connectivity between the LFPN and the right middle frontal cortex and medial frontal cortex coincided with more preserved language comprehension ability after stroke. Network connectivity analysis showed reduced LFPN connectivity as indicated by the mean network connectivity index of key regions in the LFPN of aphasic patients. The decreased LFPN connectivity in stroke patients was significantly associated with the impairment of language function in their comprehension ability. We also found significant association between recovery of comprehension ability and the mean changes in intrinsic LFPN connectivity. Our findings suggest that brain lesions may influence language comprehension by altering functional connectivity between regions and that the patterns of abnormal functional connectivity may contribute to the recovery of language deficits.

Keywords: aphasia, functional connectivity, independent component analysis, network connectivity analysis, the left frontoparietal network

\section{INTRODUCTION}

Stroke-related aphasia is a significant clinical problem persisting in one third of acute stroke patients and one fifth of chronic stroke patients (Wade et al., 1986; Berthier, 2005). Identifying the brain mechanisms underlying stroke-related aphasia is critical for understanding its prognosis and developing new therapeutic methods to treat it. In addition to local dysfunction, stroke injury to certain locations of the brain can produce specific as well as local and network dysfunction. In order to understand the influence of an individual cortical lesion, we must consider not only the loss of local neural function, but also the lesion-induced changes in the larger network interactions in the brain.

Functional segregation and integration are two major organizational principles of the human brain. An optimal brain requires a balance between local specialization and global integration of brain functional activity. However, recent work has mainly focused on defining the contribution of individual elements (e.g., inferior frontal gyrus, anterior temporal lobe) in the network. Understanding connectivity within a whole network is critical both to understanding its normal function and to explaining brain recovery (Catani et al., 2005). The function of any brain region cannot be understood in isolation but only in conjunction with the regions with which it interacts (Seghier et al., 2010).

Language is an essential higher cognitive function supported by large-scale brain networks. The superior temporal cortex (Wernicke's area) and the inferior frontal cortex (Broca's area) have been classically associated with language comprehension and production. Saur et al. (2008) identified two routes connecting the frontal and temporal language regions; a dorsal route associated with phonological processing and a ventral route associated with semantic processing. Additionally, lesion and fMRI studies (Dronkers et al., 2004; Price, 2010) have identified additional temporal, parietal, and prefrontal regions, supporting the involvement of a more extended language network (Mesulam, 1990; Turken and Dronkers, 2011). Damage to these networks (e.g., the frontal-temporal network and the frontal-parietal network) often leads to the impairment of language function, but patients frequently recover some or all of their abilities. The recovery time of aphasia varies from months to years, suggesting that recovery 
of language function following stroke is unpredictable (Pedersen et al., 1995).

In this study, we investigated the LFPN in aphasic patients by integrating an ICA approach and a network connectivity analysis method to explore how network embedding influences a region's functional role and the consequences of its being damaged. We focus on this network due to its strong association with language-cognition paradigms that are consistent with Broca's and Wernicke's areas (Smith et al., 2009). We aimed to assess the significance of functional connectivity by measuring the relationship between functional connectivity of the LFPN and performance deficits in stroke patients. More specifically, we investigated whether the degree of disruption in LFPN functional connectivity correlated with the severity of behavioral deficits at the acute stage and whether this correlation was maintained over the course of recovery.

We also aim to investigate the role of functional connectivity changes of LFPN in the recovery process following stroke. In a recent study, Park et al. (2011) reported dynamic changes in the lateralization of functional connectivity of motor networks in the first 6 months post stroke, where measures of functional connectivity at stroke onset were found to be positively correlated with motor outcomes. Studies from other groups also suggest that functional connectivity can be associated with treatment-induced behavioral changes in aphasia (Price et al., 2006; Marcotte et al., 2013). Overall, investigating the dynamic changes of functional connectivity and its association with the clinical outcomes will enhance our understanding of the relationship between human brain function and behavior (Corbetta et al., 2005; Thiebaut de Schotten et al., 2005; Sharp et al., 2010b).

\section{METHODS \\ SUBJECTS}

We recruited 14 right-handed patients [13 males, age ranging from 34 to 67 years with mean $(S D)$ : 49.4 (10.7) years] with a diagnosis of aphasia following left hemisphere stroke from the Department of Neurology at Dongzhimen Hospital over the course of 14 months (March 2012-April 2013). Experienced neurologists performed clinical assessments to confirm the diagnoses of aphasia. These assessments were based on a comprehensive evaluation, including neurological history and examination, language assessment, and structural routine MRI. The local Medical Ethical Review Board approved the protocol and we obtained written consent from all subjects prior to all experimental proceedings.

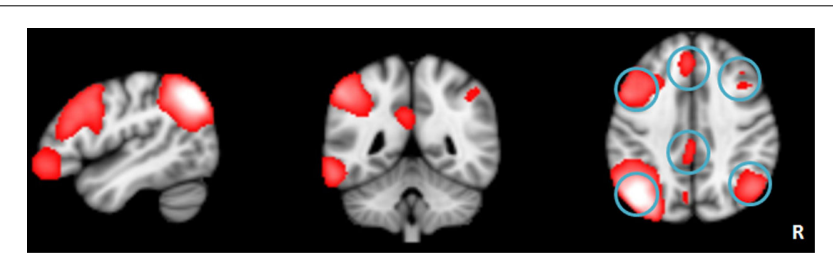

FIGURE 1 | The left frontoparietal network identified by independent component analysis.
The patients we enrolled were native Chinese speakers who were right handed, as determined by the Edinburgh Handedness Inventory (EHI) score $\geq 50$ (Oldfield, 1971). All patients had single unilateral left-hemisphere stroke and a diagnosis of aphasia based on a standardized language test from the Chinese Rehabilitation Research Center Standard Aphasia Examination (CRRCAE) (Zhang et al., 2005). All patients received a score of $\geq 2$ on the Boston Diagnostic Aphasia Examination (BDAE) severity rating scale, which indicated that they could converse about familiar topics with help from the listener, but had trouble conveying their ideas (Love and Oster, 2002) and a score of $<3$ on the modified Rankin scale, which indicated that they were moderately disabled, but able to walk without assistance (van Swieten et al., 1988). Patients were excluded from the study if they had a history of other neurological or psychiatric disorders and/or an inability to enter the MRI scanner because of non-MRI compatible prostheses.

We recruited 14 participants matched in age, gender and educational level as controls from communities near the hospital [13 males, age ranging from 34 to 67 years with mean (SD): 49.4 (10.7) years]. All subjects in the control group reported no history of neurological or psychiatric illness and were not taking regular medication.

We invited all patients to participate in two fMRI scan sessions and all healthy controls to participate in one fMRI scan session. We collected the patients' first fMRI scan (time point one) 1 month after stroke onset and the second scan (time point two) 2 months after stroke onset. We administered the CRRCAE tests before each scan.

\section{LANGUAGE ASSESSMENT}

Prior to each $\mathrm{AMRI}$ scan, we administered the CRRCAE to evaluate the degree of language impairment for each patient. This scale was developed for clinical evaluation and therapy, combining the syntactic and lexical characters in Chinese. The reliability and validity of this scale has been tested in a previous study (Zhang et al., 2005). The CRRCAE includes nine tests and 30 subtests producing a standardized score based on correct responses. The scale can test three aspects of language ability: comprehension, production, and other abilities related to language skills. In this study, we focused on the absence of comprehension and production abilities; thus, we included only comprehension (auditory and reading comprehension) and production scores (repetition, naming, and

Table 1 | The left frontoparietal network regions.

\begin{tabular}{lccc}
\hline Regions & \multicolumn{3}{c}{ MNI Coordinates } \\
\cline { 2 - 4 } & $\boldsymbol{x}$ & $\boldsymbol{y}$ & $\boldsymbol{z}$ \\
\hline Left precuneus & -40 & -70 & 46 \\
Left middle frontal gyrus & -32 & 20 & 46 \\
Right superior parietal gyrus & 42 & -66 & 50 \\
Right middle frontal gyrus & 38 & 20 & 44 \\
Posterior cingulate gyrus & -2 & -34 & 30 \\
Left medial frontal gyrus & -4 & 38 & 34
\end{tabular}




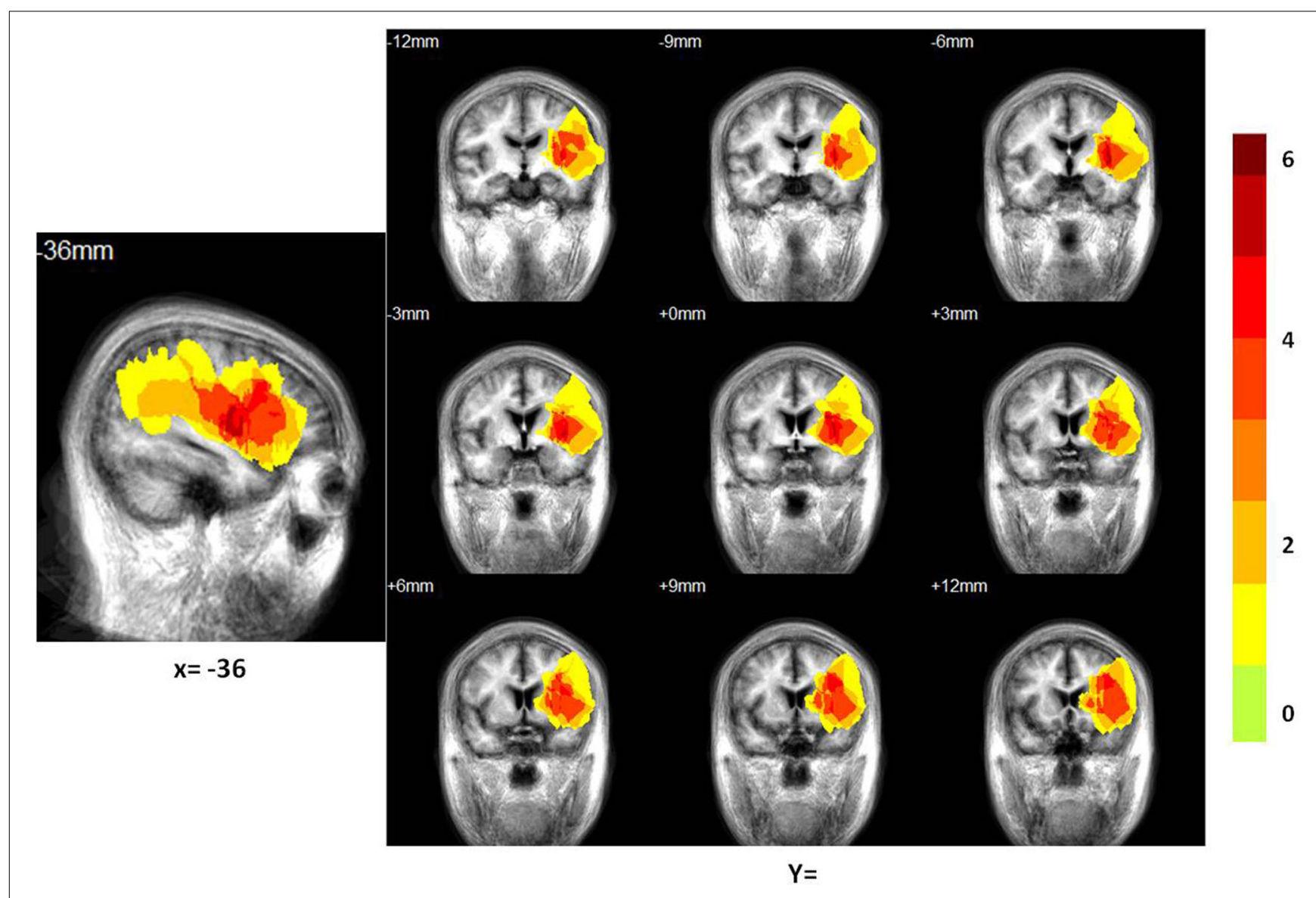

FIGURE 2 | Distribution of the lesion areas of all patients with aphasia, on the average patients' structure brain template. The intensity scale refers to the maximum number of patients with lesions at a particular voxel.

overt reading) in the correlation analysis. We used SPSS for correlation analysis. For those groups with small sample sizes $(n<10)$, we applied Spearman correlation, a nonparametric method.

\section{DATA ACQUISITION}

We performed all brain imaging on a 3T Siemens TRIO system. We used a 12-channel head coil with foam padding to restrict head motion. For resting state fMRI, we used a gradientecho echo-planar sequence sensitive to blood oxygenation leveldependent (bold) contrast $(\mathrm{TR} / \mathrm{TE}=2000 / 30 \mathrm{~ms}, \mathrm{FOV}=225 \times$ $225 \mathrm{~mm}^{2}$, flip angle $=90^{\circ}$, voxel size $=3.5 \times 3.5 \times 3.5 \mathrm{~mm}^{3}$ ). We collected 31 slices with $3.5 \mathrm{~mm}$ thickness and a $0.7 \mathrm{~mm}$ gap. Each fMRI scan lasted $6 \mathrm{~min}$ and $6 \mathrm{~s}$. The first $8 \mathrm{~s}$ were dummy scans, discarded from data analysis. Thus, we collected 179 image volumes in total. In addition, we used a highresolution T1-weighted scan [repetition time (TR)/echo time $(\mathrm{TE})=1900 / 2.13 \mathrm{~ms}$, field-of-view $(\mathrm{FOV})=256 \times 256 \mathrm{~mm}^{2}$, flip angle $=9^{\circ}$, acquired voxel size $\left.=1.0 \times 1.0 \times 1.0 \mathrm{~mm}^{3}\right]$ for anatomical localization.

\section{PATIENT LESION MAPPING}

We constructed a lesion overlap image for all 14 patients. A manually drawn outline of the lesion on the T1 image of each patient was used to overlap on the average structure image using Turtleseg (http://www.turtleseg.org/).

\section{ICA ANALYSIS OF RESTING STATE DATA}

We performed data analyses using MELODIC of FMRIB Software Library (FSL version 5.0.1; www.fmrib.ox.ac.uk/fsl) to identify large-scale patterns of temporal signal-intensity coherence, interpreted as functional connectivity, in the population of subjects (Beckmann et al., 2005). Preprocessing of functional images consisted of the removal of non-brain tissue, motion correction, temporal band-pass filtering at 0.01 to $0.1 \mathrm{~Hz}$, spatial smoothing using a $8 \mathrm{~mm}$ full-width at half-maximum Gaussian kernel, and 8-parameter nuisance signal extraction. To coregister fMRI images to a standard space, we first registered functional images to each individual's high-resolution T1 anatomical scan, and further registered them to the MNI152 template using linear affine transformations with 12 degrees of freedom.

We performed probabilistic independent component analysis (PICA) at low dimensionality (20 components) to derive the group's $(n=26)$ resting state networks. We based the network identification on their spatial similarity to functional networks described in earlier studies (Damoiseaux et al., 2006; Smith et al., 
Table 2 | Patient characteristics and subtest results of the CRRCAE at time point one and two.

\begin{tabular}{|c|c|c|c|c|c|c|c|c|c|c|}
\hline $\begin{array}{l}\text { Patient } \\
\text { number }\end{array}$ & Gender & Age & Handedness & $\begin{array}{l}\text { Aphasia } \\
\text { profile }\end{array}$ & $\begin{array}{c}\text { Comprehension } \\
\text { score }\end{array}$ & $\begin{array}{c}\text { Production } \\
\text { score }\end{array}$ & Type of stroke & Site of Iesion & $\begin{array}{c}\text { BDAE } \\
\text { severity } \\
\text { rating scale }\end{array}$ & $\begin{array}{c}\text { Modified } \\
\text { Rankin } \\
\text { scale }\end{array}$ \\
\hline 1 & $M$ & 41 & 100 & Broca's & $60 / 80$ & $80 / 90$ & Ischemia & Frontal, parietal & 4 & 0 \\
\hline 2 & M & 35 & 100 & Broca's & $70 / 75$ & $53 / 83$ & Hemorrhage & Striatocapsular & 3 & 2 \\
\hline 3 & M & 55 & 100 & Anomic & $40 / 71$ & $51 / 81$ & Ischemia & Striatocapsular & 2 & 2 \\
\hline 4 & M & 34 & 100 & Broca's & $65 / 75$ & $35 / 66$ & Hemorrhage & Striatocapsular & 3 & 2 \\
\hline 5 & M & 59 & 100 & Global & $10 / 54$ & $43 / 72$ & Ischemia & Frontal, temporal, insular & 2 & 1 \\
\hline 6 & M & 51 & 100 & Global & $32 / 72$ & $55 / 84$ & Ischemia & Frontal, parietal & 2 & 2 \\
\hline 7 & M & 58 & 90 & Global & 20/NA & 60/NA & Ischemia & Parietal, occipital & 2 & 1 \\
\hline 8 & M & 36 & 100 & Global & 33/NA & 75/NA & Ischemia & Frontal, parietal, temporal & 3 & 1 \\
\hline 9 & M & 44 & 90 & Broca's & 64/NA & 82/NA & Ischemia & Striatocapsular & 3 & 1 \\
\hline 10 & M & 63 & 100 & Broca's & 68/NA & 90/NA & Ischemia & Frontal, temporal, parietal & 4 & 0 \\
\hline 11 & M & 56 & 100 & Broca's & $75 / 80$ & $85 / 90$ & Ischemia & Striatocapsular & 4 & 0 \\
\hline 12 & $\mathrm{M}$ & 48 & 100 & Broca's & 80/NA & 90/NA & Ischemia & Striatocapsular & 4 & 0 \\
\hline 13 & M & 44 & 100 & Broca's & $70 / 80$ & $65 / 85$ & Hemorrhage & Striatocapsular & 2 & 2 \\
\hline 14 & $\mathrm{~F}$ & 67 & 100 & Global & $27 /$ NA & 50/NA & Ischemia & Frontal, temporal & 2 & 2 \\
\hline
\end{tabular}

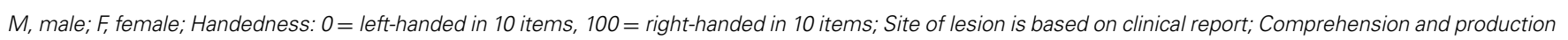

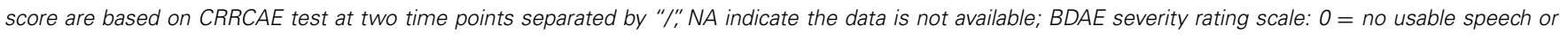
auditory comprehension, $5=$ minimal discemible speech handicaps, patient may have subjective difficulties that are not apparent to listener; Modified Rankin scale: $0=$ no symptoms at all, $6=$ dead.

2009; Biswal et al., 2010) and calculated the cross correlation between our group-level networks and the LFPN template networks derived from 1414 healthy subjects (Biswal et al., 2010). We assigned the group-derived networks that showed the highest spatial overlap with the template network to that particular functional network.

In this study, we identified the LFPN as the a priori network for further analysis. We chose this network because it is associated with language-cognition function (Smith et al., 2009). We carried out a voxel-wise comparison of the resting functional connectivity using a regression technique, referred to as the "dual-regression" approach (Filippini et al., 2009).

We used spatial maps of the group ICA in a linear model fit against each individual fMRI data set (spatial regression) to create matrices that described the temporal dynamics for each component and subject separately. We used these matrices in a linear model fit against the associated subject's fMRI data set (temporal regression) to estimate subject-specific spatial correlation maps. After this dual regression, we collected spatial maps of all subjects into single 4-dimensional files for each original independent component. We used nonparametric permutation tests to detect statistically significant differences between the groups within the boundaries of the spatial maps obtained with MELODIC. We performed all analyses with a voxel-wise cluster forming threshold of $Z>2.3$ and a corrected cluster significance threshold of $P<0.05$. We used the regions that showed significant differences between groups [spherical regions of interest (ROIs) were centered on the MNI coordinate of the cluster peaks, and with a radius of $4 \mathrm{~mm}$ ] to extract mean $z$-values from each individual spatial map, including time points one and two (FWE-corrected $P<0.05$ ) for the correlation analysis using SPSS (version 16.0; SPSS, Chicago, IL,
USA). Then we performed correlation analyses to assess association between the ROIs within the LFPN and language test results.

\section{NETWORK CONNECTIVITY ANALYSIS OF LEFT FRONTOPARIETAL NETWORK}

To better understand the impact of network connectivity differences on whole-brain intrinsic connectivity, we employed network connectivity analysis to LFPN changes. We used standard image processing methods with SPM8 (http://www.fil. ion.ucl.ac.uk/spm) and the conn toolbox (http://www.nitrc.org/ projects/conn) for functional connectivity and network connectivity analysis. Our pre-processing steps included correcting for motion, coregistering with the anatomic scan, normalizing into the Montreal Neurological Institute space, resampling at $2 \mathrm{~mm}^{3}$, and smoothing with a Gaussian kernel of $6 \mathrm{~mm}^{3}$ full-width at half maximum. We extracted the bold time series data for six ROIs within the LFPN obtained from the ICA analysis mentioned above. The ROIs were derived from the LFPN (centered on the MNI coordinate of the cluster peaks, and with a radius of $4 \mathrm{~mm}$ ) (Figure 1). Prior connectivity studies have employed similar approaches to the investigation of the default mode network (DMN) (Dosenbach et al., 2007; Fair et al., 2008; Posner et al., 2013). The LFPN ROIs and their coordinates are delineated in Table 1. We correlated the time series data for each ROI region by region for each subject, producing a single $6 \times 6$ correlation matrix for each subject. We calculated the mean index of the LFPN by reducing each subject's $6 \times 6$ correlation matrix from the overall mean into a single variable that indexed the global connectivity for the LFPN including all edges. We compared the mean network connectivity index across the patient and control groups using a two-sample $t$-test. We also performed correlation 


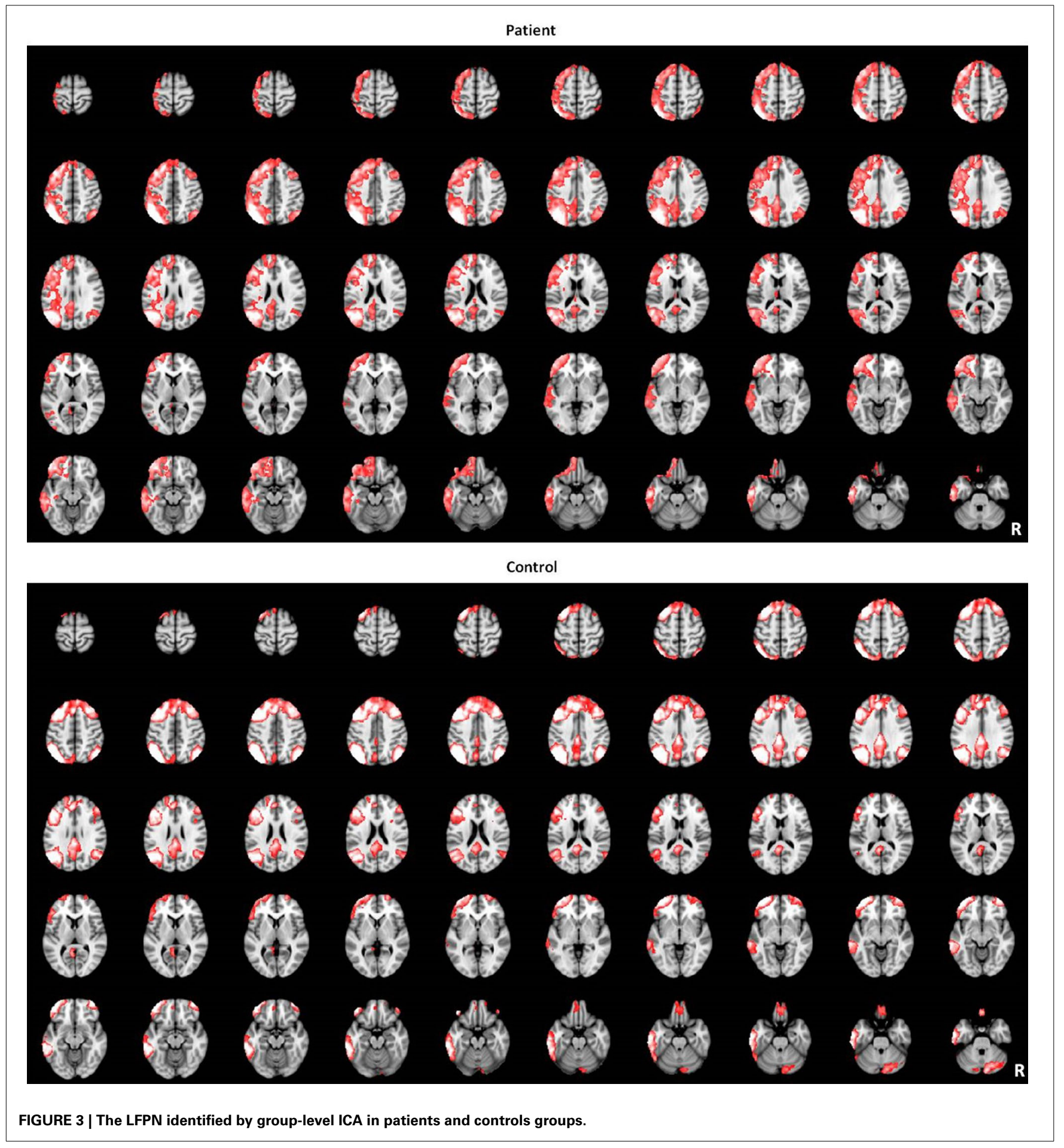

analyses to assess the relationship between the mean connectivity of the LFPN and the language test results.

\section{RESULTS}

\section{DEMOGRAPHICS AND LANGUAGE PERFORMANCE}

The locations of the patients' infarcts are shown in Figure 2. Of the total 28 subjects (14 patients) enrolled in the study, we dropped one control subject due to technical issues in brain structure and one patient due to failure in the pre-processing of the ICA and network connectivity analysis. We included 13 patients and 13 controls in the ICA and network connectivity analyses. Of all 13 patients, eight patients completed the second scan. Six patients could not participate in the second scan due to their inability to return to the hospital. For characteristics of 


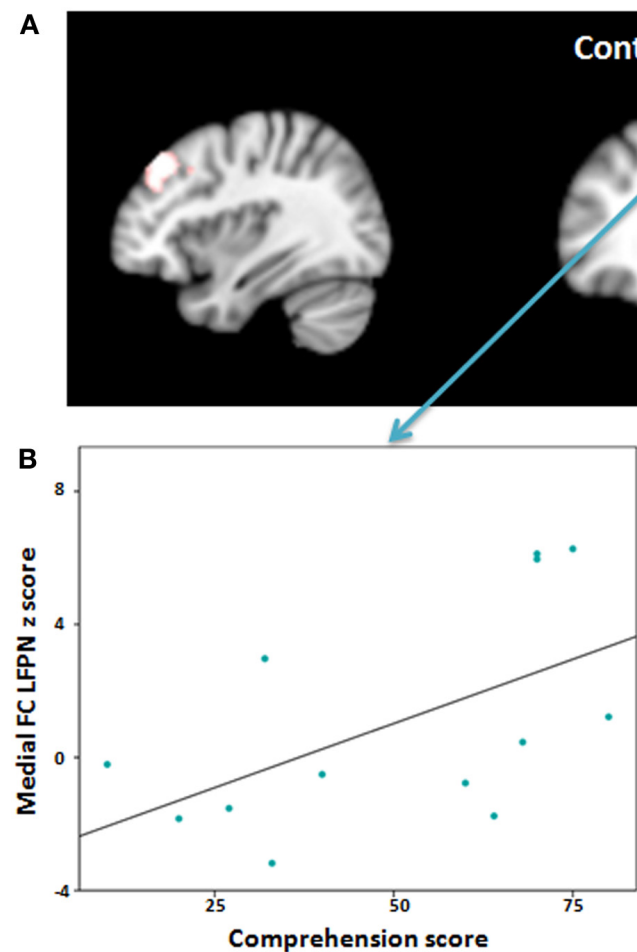

FIGURE 4 | (A) Red-white heat map represents results of decreased functional connectivity within left frontoparietal network in patients compared with controls. (B,C) Scatter plots depict the relationship between abnormal regions functional connectivity and severity of comprehension deficit at time point one in patient group. FC, Frontal cortex; LFPN, left frontoparietal network. these patients and scores from all subtests of the CRRCAE, see Table 2.

The comprehension subtest scores that we calculated consist of the combined auditory and reading comprehension scores of each subject and the production subtest score consists of the combined repetition, naming, and overt reading scores of each subject. At time point one $(n=14)$, the scores on the comprehension and production subtests were 51 (23) [mean (SD)] and 65 (18), respectively. At time point two $(n=8)$, the scores for comprehension and production were 73 (9) and 81 (8), respectively. The nonparametric rank test showed that at time point two, there was a significant increase in the comprehension $(z=-2.527$, $P=0.012)$ and production $(z=-2.527, P=0.012)$ score as compared to that at time point one. The average improvement of the CRRCAE scores of the patients on the comprehension and production subtests between the two time points were 21 (16) and 23 (10), respectively.

All patients received conventional stroke treatment, which involved a 30-min language therapy session that involved listening comprehension, reading comprehension, and verbal production training at least three times per week throughout the entire period of observation.

\section{RESULTS FROM ICA ANALYSIS}

The LFPN that we obtained from our cohort of subjects includes the bilateral parietal cortex, bilateral frontal cortex, medial frontal cortex and posterior cingulate cortex
Table 3 | Decreased FC clusters in aphasic patients compared with controls ( $P<0.05$, using FWE correction at cluster level).

\begin{tabular}{|c|c|c|c|c|c|c|}
\hline \multirow[t]{2}{*}{ Contrast } & \multirow[t]{2}{*}{ Brain region } & \multirow{2}{*}{$\begin{array}{l}\text { Cluster } \\
\text { size }\end{array}$} & \multicolumn{3}{|c|}{ MNI coordinates } & \multirow[t]{2}{*}{ z-value } \\
\hline & & & $\boldsymbol{x}$ & $y$ & $z$ & \\
\hline \multirow[t]{3}{*}{$\begin{array}{l}\text { Control > } \\
\text { patient }\end{array}$} & $\begin{array}{l}\text { Right middle } \\
\text { frontal cortex }\end{array}$ & 199 & 36 & 34 & 42 & 3.82 \\
\hline & $\begin{array}{l}\text { Medial frontal } \\
\text { cortex }\end{array}$ & 149 & 0 & 30 & 42 & 3.8 \\
\hline & $\begin{array}{l}\text { Right inferior } \\
\text { frontal cortex }\end{array}$ & 5 & 58 & 30 & 4 & 3.45 \\
\hline
\end{tabular}

(Figure 1). We obtained 20 spatial and temporal components from the ICA analysis and noted that the results from previous ICA analyses support the selection of the LFPN (Smith et al., 2009; Biswal et al., 2010). The grouplevel ICA was performed separately in patients and controls (Figure 3).

When we compared the patients to the matched healthy controls, we found significantly reduced functional connectivity between the LFPN and the right middle frontal cortex, the medial frontal cortex, and the right inferior frontal cortex (Figure 4A, Table 3) in aphasic patients. No regions showed significantly increased functional connectivity in patients as compared to controls. 


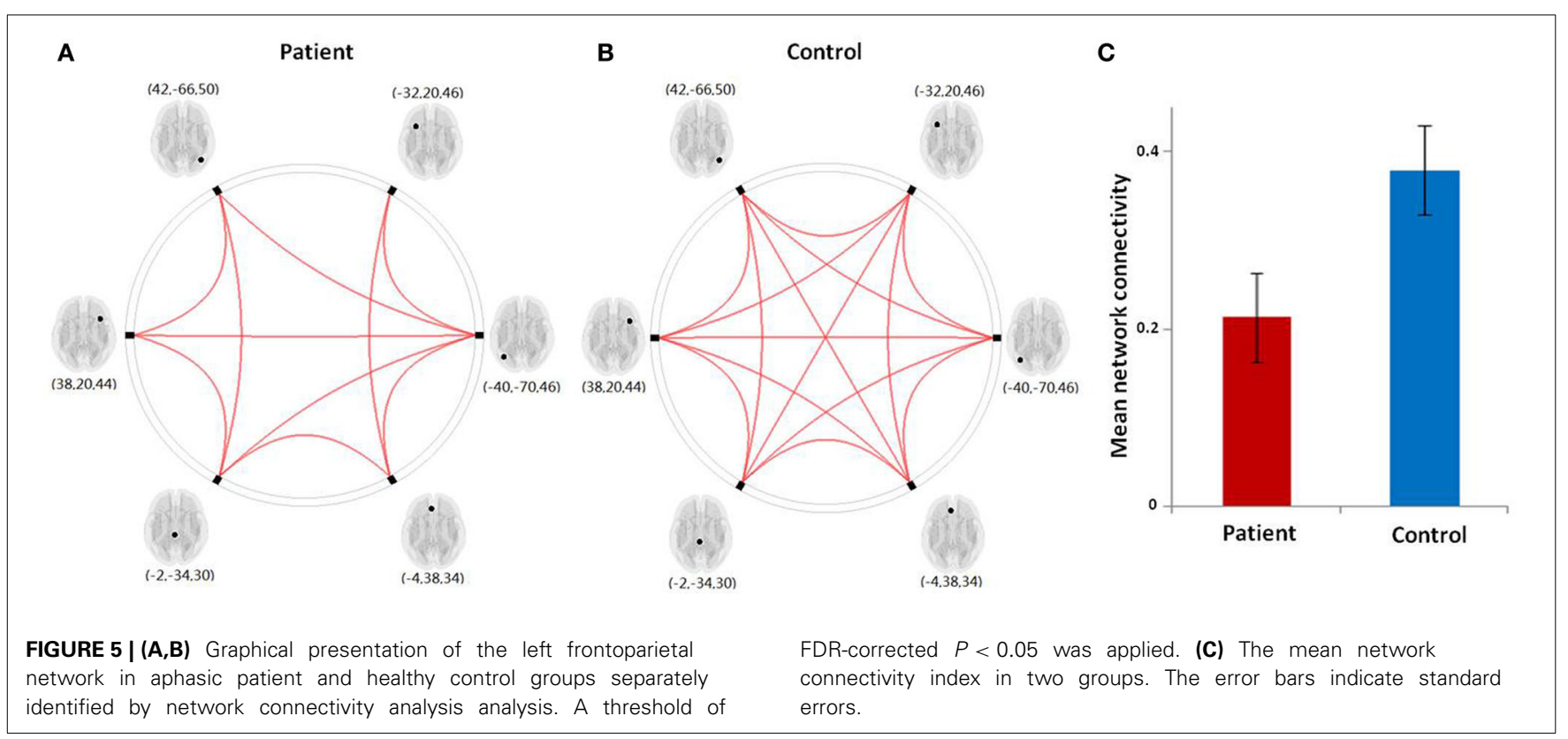

To explore the association between language impairment and lower regional functional connectivity within the LFPN, we applied a Pearson correlation between the regions that showed significant differences in the language production and comprehension scores separately at time point one. The results showed a significant association between the medial frontal cortex and comprehension score $(r=0.555, P=0.049$; Figure 4B) and strong correlation between the right middle frontal cortex and comprehension score $(r=0.781, P=0.002$; Figure 4C), such that lower intrinsic functional connectivity between the LFPN and right middle frontal cortex and medial frontal cortex coincided with greater impairment of comprehension ability in aphasic patients. To test whether this correlation was maintained over the course of recovery, we also measured the association between LFPN functional connectivity and the above two regions and the comprehension score at time point two using a Spearman correlation for eight patients. Our results showed a marginally significant association between the LFPN and the right middle frontal cortex connectivity and comprehension score $(r=0.728$, $P=0.064)$. We found no significant association between these three regions and production score.

\section{RESULTS FROM NETWORK CONNECTIVITY ANALYSIS}

The results from our network connectivity analysis suggest that patients and controls are associated with two different sets of network connectivity. At time point one, the network of the controls was considerably more intact than that of the patients (Figures 5A,B). The network showed a different pattern at time point one as compared to time point two (Figures 6A,B). At time point one, we observed significantly weaker LFPN connectivity in patients as compared to controls (mean network connectivity index of patients: 0.213 and controls: $0.379 ; t=3.104, P=0.005$ ) (Figure 5C).

The Pearson correlation analysis showed a significant association between mean LFPN connectivity and comprehension score at time point one $(r=0.781, P=0.002)$ in aphasic patients, indicating that decreased LFPN connectivity coincided with more severe the loss of comprehension ability.

For the eight patients that completed the two fMRI scans, the Spearman correlation analysis showed that the change in mean network connectivity index was marginally associated with the improvement of comprehension ability $(r=0.655, P=0.078$; Figure 6C). That is, patients who exhibited the highest level of comprehension improvement also showed the highest increase in mean LFPN network connectivity.

\section{DISCUSSION}

In this study, we integrated ICA and network connectivity analysis methods to investigate the association between resting state functional connectivity and language function in aphasic patients. We found reduced functional connectivity between the LFPN and the right middle frontal cortex and medial frontal cortex in aphasic patients. Stronger functional connectivity coincided with more preserved language comprehension ability after stroke. This strengthening in connectivity could be maintained over the course of recovery in the right middle frontal cortex. In addition, we found reduced LFPN connectivity in aphasic patients, as indicated by the mean network connectivity index of key regions in the LFPN. We associated the decreased LFPN connectivity with the impairment of language function in the comprehension ability of stroke patients. We also found significant association between the recovery of comprehension ability and the mean improvement in intrinsic LFPN connectivity.

Speech comprehension ability reflects a complex cognitive process, including attention, working memory, comprehension monitoring, and strategic behavior. In aphasic patients, we found decreased functional connectivity between the LFPN and the right middle frontal cortex, the medial frontal cortex, and the right inferior frontal cortex. Previous studies found that the medial prefrontal cortex is activated during task switching and 

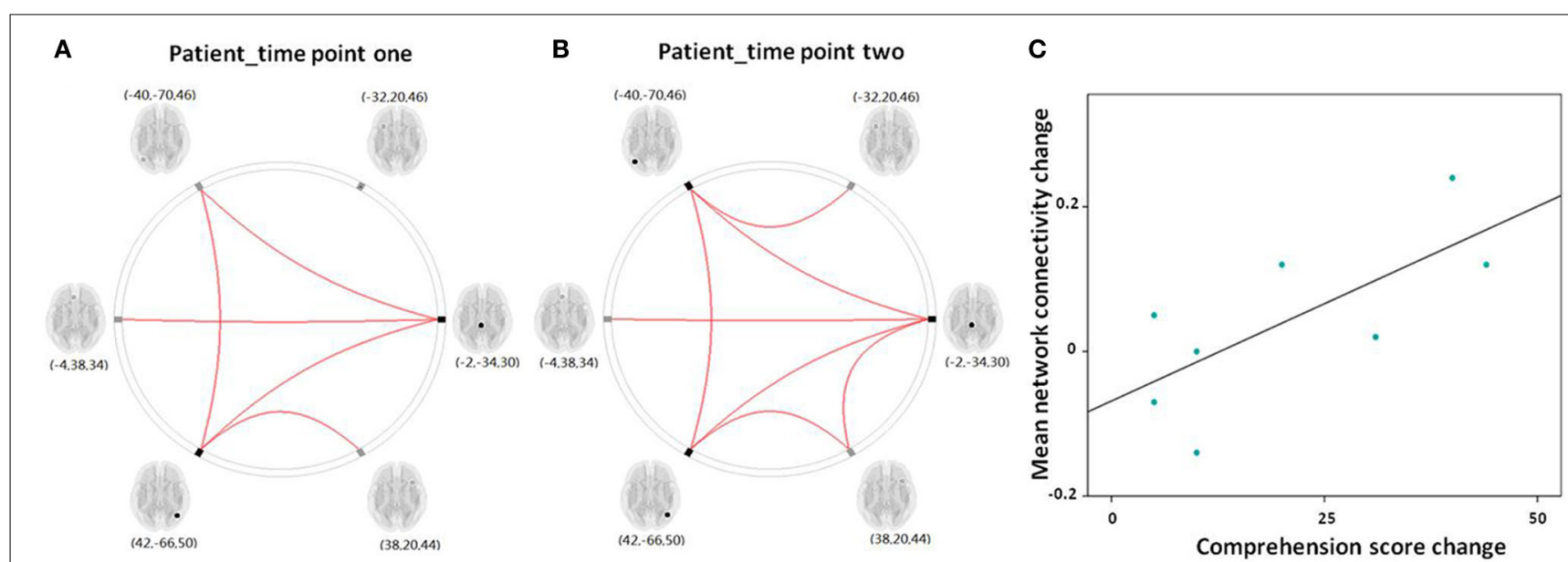

FIGURE 6 | (A,B) Graphical presentation of the left frontoparietal network in aphasic patient in time point one and two separately identified by network connectivity analysis analysis. A threshold of FDR-corrected $P<0.05$ was applied. (C) Scatter plots depict the relationship between the mean left frontoparietal network connectivity change and the comprehension score change in patient group between time point one and two. performance monitoring and/or adjustments (DiGirolamo et al., 2001; Rushworth et al., 2002; Ridderinkhof et al., 2004; Wager et al., 2004; Crone et al., 2006). More specifically, using resting state connectivity over more than 1000 subjects, investigators have found that both subdivisions in the medial frontal cortex and posterior cingulate cortex are strongly connected to the inferior parietal lobe, a key region in language processing (Lambon Ralph, 2010). Historically, investigators have defined the dorsal, rather than the ventral, medial frontal cortex by its connection to the inferior parietal cortex (Luciana, 2001). This was not part of the LFPN we defined in this study. Our results showed that LFPN showed a significant association with dorsal medial prefrontal cortex, we speculate these regions may interact rather strongly during task-related processes (Tomasi and Volkow, 2012). Results from previous studies have also suggested that the medial prefrontal cortex is involved in coherence processing in language comprehension (i.e., establishing the pragmatic connection between successively presented sentences) (Ferstl and von Cramon, 2002). Our results support the idea that the medial prefrontal cortex plays an important role in language processing.

The left middle frontal cortex and the inferior frontal cortex are also critical components of language processing. Investigators have interpreted the involvement of the inferior frontal cortex in aphasic patients as reflecting the impairment of a working memory system for semantic information, whereas the middle frontal cortex has been attributed to deficits in the general cognitive control process (Turken and Dronkers, 2011). We believe that comprehension ability in patients could reflect the semantic cognition process. Results from a previous study suggest that lesions located in the left hemisphere after stroke can cause the right hemisphere to selectively contribute to the reorganization of language (Crinion and Price, 2005). This may be due to the disinhibition of the right hemisphere in the presence of left hemisphere lesions. Other studies have suggested that a relationship exists between lesion size and the success of hemispheric transfer, where larger lesions may result in a complete transfer of functions to the contralateral hemisphere. Conversely, in the presence of a smaller lesion, intact areas of the damaged hemisphere may inhibit complete transfer (Grafman, 2000). We speculate that in our study, the lesions of patients may have not been large enough, so that the right hemisphere was still inhibited by the intact remains in the left hemisphere. This may explain why we found decreased functional connectivity in the homologous parts of these two regions in the LFPN. We also found decreased connectivity in the middle frontal cortex to be associated with cognitive function and patients maintained this correlation over the course of a 1-month recovery. This finding is consistent with prior literature on the reorganization of language recovery after stroke.

Overall, we have found a multitude of evidence supporting our finding that the LFPN may be especially relevant to understanding cognitive impairment in aphasic patients. Considering the complexity of language processing, which includes semantic, lexical and phonological levels, motor programming, and access to visual and memory representations in oral naming, the network analysis approach seems particularly suitable for characterizing post-stroke recovery. Under the assumption that disconnection between the distal frontoparietal areas may underlie primary cognitive deficits, recent fMRI studies have explored the frontoparietal network and its association with performance in attention and working memory tasks (Sharp et al., 2010a,b). In our study, aphasic patients showed a significant decrease in mean LFPN connectivity as compared to controls and the magnitude of this decrease was correlated with language comprehension ability. This result may suggest that there was disrupted functional connectivity in the LFPN, which induced cognitive deficits at the acute stage of lesion to the left hemisphere.

After 1 month of recovery following stroke, mean LFPN connectivity seems to be associated with the recovery of language comprehension function. Increasing LFPN connectivity is likely to be a natural, intrinsic, and plastic neural mechanism for increased cognitive function and can be regarded as 
early language recovery. Specifically, investigators have found that increased frontoparietal integration during language task processing in patients who are in recovery following aphasia can be associated with the recovery of cognitive function (Sharp et al., 2010b). Reorganization of the functional network is essential to the recovery of language function. We found that increased LFPN connectivity corresponded with greater improvement in language function after 1 month of recovery following stroke. This result suggests that promoting this connectivity should be an important target for future research aimed at restoring language deficits.

Our study was not without limitations. The patients completing two fMRI scans received a conventional therapy, so we remain unsure about whether to attribute the changes in network functional connectivity to the medication or the natural recovery of language function. Additionally, many aphasic patients had multifocal brain lesions in the left hemisphere, which may have potentially complicated the interpretation of our results. Brain damage to the left hemisphere regions - the frontal or parietal cortex, this may cause functional network abnormalities that induce language function deficit. In all 13 patients, only two patients had wide lesions spreading to the frontal and parietal cortices and no more than three patients had a lesion in the same area of the left frontoparietal network. Still, it is reasonable to believe that the function of the LFPN was preserved in our sample of patients. Thus, studies with larger sample sizes that only enroll patients with unifocal lesions are necessary to verify our findings in the future.

In summary, we found functional connectivity abnormalities in the LFPN in aphasic patients. Our results suggest that brain lesions may influence language comprehension ability by causing impairment of both functionality in affected regions and functional connectivity with other regions. Identifying the patterns of abnormal functional connectivity may contribute to therapies that enhance the recovery of language deficits and cognitive function following stroke.

\section{ACKNOWLEDGMENTS}

We deeply thank Hsiao-Ying Wey, Xiaoyan Chen, and Javeria Ali Hashmi in the Department of Psychiatry, Massachusetts General Hospital for their valuable help in data analysis. This work was supported by the National Science Foundation of China (Grant No. 81072768), New Century Excellent Talents in University of Ministry of Education of China (Grant No. NCET-11-0603), Chinese Medicine Clinical Evaluation Research Team in Brain Disease of Beijing University of Chinese Medicine (Grant No. 2011-CXTD-22).

\section{REFERENCES}

Beckmann, C. F., DeLuca, M., Devlin, J. T., and Smith, S. M. (2005). Investigations into resting-state connectivity using independent component analysis. Philos. Trans. R. Soc. Lond. B Biol. Sci. 360, 1001-1013. doi: 10.1098/rstb. 2005.1634

Berthier, M. L. (2005). Poststroke aphasia: epidemiology, pathophysiology and treatment. Drugs Aging 22, 163-182. doi: 10.2165/00002512-200522020-00006

Biswal, B. B., Mennes, M., Zuo, X. N., Gohel, S., Kelly, C., and Smith, S. M. (2010). Toward discovery science of human brain function. Proc. Natl. Acad. Sci. U.S.A. 107, 4734-4739. doi: 10.1073/pnas.0911855107

Catani, M., Jones, D. K., and ffytche, D. H. (2005). Perisylvian language networks of the human brain. Ann. Neurol. 57, 8-16. doi: 10.1002/ana.20319
Corbetta, M., Kincade, M. J., Lewis, C., Snyder, A. Z., and Sapir, A. (2005). Neural basis and recovery of spatial attention deficits in spatial neglect. Nat. Neurosci. 8, 1603-1610. doi: 10.1038/nn1574

Crinion, J., and Price, C. J. (2005). Right anterior superior temporal activation predicts auditory sentence comprehension following aphasic stroke. Brain 128(pt 12), 2858-2871. doi: 10.1093/brain/awh659

Crone, E. A., Wendelken, C., Donohue, S. E., and Bunge, S. A. (2006). Neural evidence for dissociable components of task-switching. Cereb. Cortex 16, 475-486. doi: 10.1093/cercor/bhi127

Damoiseaux, J. S., Rombouts, S. A., Barkhof, F., Scheltens, P., Stam, C. J., and Smith, S. M. (2006). Consistent resting-state networks across healthy subjects. Proc. Natl. Acad. Sci. U.S.A. 103, 13848-13853. doi: 10.1073/pnas.0601 417103

DiGirolamo, G. J., Kramer, A. F., Barad, V., Cepeda, N. J., Weissman, D. H., and Milham, M. P. (2001). General and task-specific frontal lobe recruitment in older adults during executive processes: a fMRI investigation of task-switching. Neuroreport 12, 2065-2071. doi: 10.1097/00001756-20010703000054

Dosenbach, N. U., Fair, D. A., Miezin, F. M., Cohen, A. L., Wenger, K. K., and Dosenbach, R. A. (2007). Distinct brain networks for adaptive and stable task control in humans. Proc. Natl. Acad. Sci. U.S.A. 104, 11073-11078. doi: 10.1073/pnas.0704320104

Dronkers, N. F., Wilkins, D. P., Van Valin, R. D. Jr., Redfern, B. B., and Jaeger, J. J. (2004). Lesion analysis of the brain areas involved in language comprehension. Cognition 92, 145-177. doi: 10.1016/j.cognition.2003.11.002

Fair, D. A., Cohen, A. L., Dosenbach, N. U., Church, J. A., Miezin, F. M., and Barch, D. M. (2008). The maturing architecture of the brain's default network. Proc. Natl. Acad. Sci. U.S.A. 105, 4028-4032. doi: 10.1073/pnas.0800376105

Ferstl, E. C., and von Cramon, D. Y. (2002). What does the frontomedian cortex contribute to language processing: coherence or theory of mind? Neuroimage 17, 1599-1612. doi: 10.1006/nimg.2002.1247

Filippini, N., MacIntosh, B. J., Hough, M. G., Goodwin, G. M., Frisoni, G. B., and Smith, S. M. (2009). Distinct patterns of brain activity in young carriers of the APOE-epsilon4 allele. Proc. Natl. Acad. Sci. U.S.A. 106, 7209-7214. doi: 10.1073/pnas.0811879106

Grafman, J. (2000). Conceptualizing functional neuroplasticity. J. Commun. Disord. 33, 345-355; quiz 355-346. doi: 10.1016/S0021-9924(00)00030-7

Lambon Ralph, M. A. (2010). Measuring language recovery in the underlying largescale neural network: pulling together in the face of adversity. Ann. Neurol. 68, 570-572. doi: 10.1002/ana.22213

Love, T., and Oster, E. (2002). On the categorization of aphasic typologies: the SOAP (a test of syntactic complexity). J. Psycholinguist. Res. 31, 503-529. doi: 10.1023/A:1021208903394

Luciana, M. (2001). Handbook of Developmental Cognitive Neuroscience. Cambridge, MA: MIT Press.

Marcotte, K., Perlbarg, V., Marrelec, G., Benali, H., and Ansaldo, A. I. (2013). Default-mode network functional connectivity in aphasia: therapy-induced neuroplasticity. Brain Lang. 124, 45-55. doi: 10.1016/j.bandl.2012.11.004

Mesulam, M. M. (1990). Large-scale neurocognitive networks and distributed processing for attention, language, and memory. Ann. Neurol. 28, 597-613. doi: 10.1002/ana.410280502

Oldfield, R. C. (1971). The assessment and analysis of handedness: the Edinburgh inventory. Neuropsychologia 9, 97-113. doi: 10.1016/0028-3932(71)90067-4

Park, C. H., Chang, W. H., Ohn, S. H., Kim, S. T., Bang, O. Y., and PascualLeone, A. (2011). Longitudinal changes of resting-state functional connectivity during motor recovery after stroke. Stroke 42, 1357-1362. doi: 10.1161/STROKEAHA.110.596155

Pedersen, P. M., Jorgensen, H. S., Nakayama, H., Raaschou, H. O., and Olsen, T. S. (1995). Aphasia in acute stroke: incidence, determinants, and recovery. Ann. Neurol. 38, 659-666. doi: 10.1002/ana.410380416

Posner, J., Hellerstein, D. J., Gat, I., Mechling, A., Klahr, K., and Wang, Z. (2013). Antidepressants normalize the default mode network in patients with dysthymia. JAMA Psychiatry 70, 373-382. doi: 10.1001/jamapsychiatry.2013.455

Price, C. J. (2010). The anatomy of language: a review of $100 \mathrm{fMRI}$ studies published in 2009. Ann. N.Y. Acad. Sci. 1191, 62-88. doi: 10.1111/j.17496632.2010.05444.x

Price, C. J., Crinion, J., and Friston, K. J. (2006). Design and analysis of fMRI studies with neurologically impaired patients. J. Magn. Reson. Imaging 23, 816-826. doi: 10.1002/jmri.20580 
Ridderinkhof, K. R., Ullsperger, M., Crone, E. A., and Nieuwenhuis, S. (2004). The role of the medial frontal cortex in cognitive control. Science 306, 443-447. doi: 10.1126/science. 1100301

Rushworth, M. F., Hadland, K. A., Paus, T., and Sipila, P. K. (2002). Role of the human medial frontal cortex in task switching: a combined fMRI and TMS study. J. Neurophysiol. 87, 2577-2592. doi: 10.1152/jn.00812.2001

Saur, D., Kreher, B. W., Schnell, S., Kummerer, D., Kellmeyer, P., and Vry, M. S. (2008). Ventral and dorsal pathways for language. Proc. Natl. Acad. Sci. U.S.A. 105, 18035-18040. doi: 10.1073/pnas.0805234105

Seghier, M. L., Zeidman, P., Neufeld, N. H., Leff, A. P., and Price, C. J. (2010). Identifying abnormal connectivity in patients using dynamic causal modeling of FMRI responses. Front. Syst. Neurosci. 4:142. doi: 10.3389/fnsys.2010. 00142

Sharp, D. J., Awad, M., Warren, J. E., Wise, R. J., Vigliocco, G., and Scott, S. K. (2010a). The neural response to changing semantic and perceptual complexity during language processing. Hum. Brain Mapp. 31, 365-377. doi: 10.1002/hbm.20871

Sharp, D. J., Turkheimer, F. E., Bose, S. K., Scott, S. K., and Wise, R. J. (2010b). Increased frontoparietal integration after stroke and cognitive recovery. Ann. Neurol. 68, 753-756. doi: 10.1002/ana.21866

Smith, S. M., Fox, P. T., Miller, K. L., Glahn, D. C., Fox, P. M., and Mackay, C. E. (2009). Correspondence of the brain's functional architecture during activation and rest. Proc. Natl. Acad. Sci. U.S.A. 106, 13040-13045. doi: 10.1073/pnas.0905267106

Thiebaut de Schotten, M., Urbanski, M., Duffau, H., Volle, E., Levy, R., and Dubois, B. (2005). Direct evidence for a parietal-frontal pathway subserving spatial awareness in humans. Science 309, 2226-2228. doi: 10.1126/science. 1116251

Tomasi, D., and Volkow, N. D. (2012). Resting functional connectivity of language networks: characterization and reproducibility. Mol. Psychiatry 17, 841-854. doi: $10.1038 / \mathrm{mp} .2011 .177$
Turken, A. U., and Dronkers, N. F. (2011). The neural architecture of the language comprehension network: converging evidence from lesion and connectivity analyses. Front. Syst. Neurosci. 5:1. doi: 10.3389/fnsys.2011.00001

van Swieten, J. C., Koudstaal, P. J., Visser, M. C., Schouten, H. J., and van Gijn, J. (1988). Interobserver agreement for the assessment of handicap in stroke patients. Stroke 19, 604-607. doi: 10.1161/01.STR.19.5.604

Wade, D. T., Hewer, R. L., David, R. M., and Enderby, P. M. (1986). Aphasia after stroke: natural history and associated deficits. J. Neurol. Neurosurg. Psychiatry 49, 11-16. doi: 10.1136/jnnp.49.1.11

Wager, T. D., Jonides, J., and Reading, S. (2004). Neuroimaging studies of shifting attention: a meta-analysis. Neuroimage 22, 1679-1693. doi: 10.1016/j.neuroimage.2004.03.052

Zhang, Q. S., Ji, S. R., and Li, S. L. (2005). Reliability and validity of Chinese rehabilitation research center standard aphasia examination. Chin. J. Rehabil. Theory Pract. 11, 703-705 (in chinese).

Conflict of Interest Statement: The authors declare that the research was conducted in the absence of any commercial or financial relationships that could be construed as a potential conflict of interest.

Received: 03 February 2014; accepted: 18 April 2014; published online: 12 May 2014. Citation: Zhu D, Chang J, Freeman S, Tan Z, Xiao J, Gao Y and Kong J (2014) Changes of functional connectivity in the left frontoparietal network following aphasic stroke. Front. Behav. Neurosci. 8:167. doi: 10.3389/fnbeh.2014.00167

This article was submitted to the journal Frontiers in Behavioral Neuroscience.

Copyright (C) 2014 Zhu, Chang, Freeman, Tan, Xiao, Gao and Kong. This is an openaccess article distributed under the terms of the Creative Commons Attribution License (CC BY). The use, distribution or reproduction in other forums is permitted, provided the original author(s) or licensor are credited and that the original publication in this journal is cited, in accordance with accepted academic practice. No use, distribution or reproduction is permitted which does not comply with these terms. 